\title{
High sugar intake via the renin-angiotensin system blunts the baroreceptor reflex in adult rats that were perinatally depleted of taurine
}

\author{
Atcharaporn Thaeomor ${ }^{1}$, J Michael Wyss ${ }^{2}$, Dusit Jirakulsomchok', Sanya Roysommuti ${ }^{1 *}$ \\ From $17^{\text {th }}$ International Meeting of Taurine \\ Fort Lauderdale, FL, USA. 14-19 December 2009
}

\begin{abstract}
Perinatal taurine depletion leads to several physiological impairments in adult life, in part, due to taurine's effects on the renin-angiotensin system, a crucial regulator of growth and differentiation during early life. The present study tests the hypothesis that perinatal taurine depletion predisposes adult female rats to impaired baroreceptor control of arterial pressure by altering the renin-angiotensin system. Female Sprague Dawley (SD) rats were fed normal rat chow and from conception to weaning drank 3\% beta-alanine in water (taurine depletion, TD) or water alone (Control, C). Female offspring ate a normal rat chow and drank water with (G) or without (W) 5\% glucose throughout the experiment. To test the possible role of the renin-angiotensin system, $50 \%$ of the rats received captopril (an angiotensin converting enzyme inhibitor, $400 \mathrm{mg} / \mathrm{L}$ ) from 7 days before parameter measurements until the end of experiment. At 7-8 weeks of age, arterial pressure, heart rate, baroreflex control of heart rate and renal nerve activity were studied in either conscious, freely moving or anesthetized rats. Perinatal taurine depletion did not alter resting mean arterial pressure or heart rate in the adult female offspring that received either high or normal sugar intake. Captopril treatment slightly decreased mean arterial pressure but not heart rate in all groups. Compared to controls, only the TDG rats displayed blunted baroreflex responses. Captopril treatment normalized baroreflex sensitivity in TDG. The present data indicate that in perinatal taurine depleted female rats, the reninangiotensin system underlines the ability of high sugar intake to blunt baroreceptor responses.
\end{abstract}

\section{Introduction}

Taurine is present at a high concentration in many organs including brain, heart, kidneys, and reproductive organs. Taurine content in these organs is highest during the perinatal period, and it modestly declines with advancing age [1]. Several lines of evidence indicate that in addition to other aspects of the perinatal environment (e.g., nutrition and hormones), taurine contributes to programming adult function and diseases susceptibility, especially in relation to the cardiovascular system [2]. Poor nutrition in early life can lead to obesity, diabetes mellitus, hypertension and coronary heart diseases in adults [3], and via epigenetic mechanisms, these can

\footnotetext{
* Correspondence: sanya@kku.ac.th

'Department of Physiology Faculty of Medicine, Khon Kaen University, Khon Kaen 40002, Thailand

Full list of author information is available at the end of the article
}

transfer to the next generation. In addition, perinatal inhibition of the renin-angiotensin system impairs renal function [4,5] and induces salt-sensitive hypertension in normotensive animals but prevents hypertension in spontaneously hypertensive rats [6,7].

Taurine supplementation either during perinatal period or during young adult life prevents hypertension in adult spontaneously hypertensive rats, partly by its antioxidant action [8]. Exposure to excess taurine in perinatal life influences growth and autonomic nervous system control of arterial pressure in adult male rats $[9,10]$. Renal hemodynamics are sensitive to perinatal taurine action [11], as demonstrated by the finding that taurine transporter knockout mice display several abnormalities in renal structure and function in adult life $[12,13]$. In addition, perinatal taurine depletion heightens sugarinduced hypertension in the adult male offspring [10], 
and this effect impairs renal function prior to the appearance of hypertension and diabetes mellitus $[9,14]$. Renin-angiotensin system overactivity underlines this phenomenon.

The present study tests the hypothesis that perinatal taurine depletion via renin-angiotensin mechanisms impairs baroreceptor reflex control of arterial pressure in adult female rats. Hyperinsulinemia and insulin resistance are also investigated.

\section{Materials and methods Animal preparation}

Sprague Dawley (SD) rats were bred from the animal unit of Faculty of Medicine, Khon Kaen University and maintained at constant humidity $(60 \pm 5 \%)$, temperature $\left(24 \pm 1{ }^{\circ} \mathrm{C}\right)$, and light cycle (0600-1800 h). Female SD rats were fed normal rat chow and drank $3 \%$ beta-alanine in water (taurine depletion, TD) or water alone (Control, C) from conception to weaning. Female offspring were then fed the normal rat chow with either $5 \%$ glucose in tap water (G) or tap water alone (W) throughout the experiment. To inhibit the renin-angiotensin system, captopril (an angiotensin converting enzyme inhibitor, $400 \mathrm{mg} / \mathrm{L}$ ) was administered in drinking water of $50 \%$ of the rats in each group from 7 days before initial testing until the end of experiment. At 7-8 weeks of age, arterial pressure and heart rate and baroreflex control of heart rate and renal nerve activity were tested in either conscious, freely moving or anesthetized (thiopental sodium, $50 \mathrm{mg} / \mathrm{kg}$, i.p.) rats. All experimental procedures were preapproved by the Khon Kaen University Animal Care and Use Committee and were conducted in accordance with the National Institutes of Health guidelines.

\section{Experimental protocol}

At the time of study, all female rats were anesthetized by thiopental sodium and then were implanted with femoral arterial and venous catheters. Two or three days later, after an overnight fast, arterial blood samples $(1.0 \mathrm{ml}$ each) were taken from conscious rats and analyzed for $\mathrm{Na}, \mathrm{K}$, hematocrit, blood urea nitrogen, creatinine, insulin, and fasting blood sugar determinations. Blood volumes were immediately replaced with equal volumes of donor blood from rats of same treatment. Twenty-four hours later, arterial pressure was continuously recorded (BIOPAC, Goleta, CA) in conscious rats before and during infusion of phenylephrine (PE; to increase arterial pressure) or sodium nitroprusside (SNP; to decrease arterial pressure). One-day later, female rats were anesthetized with thiopental sodium and tracheostomized, and arterial pulse was recorded continuously for assessment of baroreflex control of renal nerve activity. Body temperature was servo- control at $37 \pm 0.5^{\circ} \mathrm{C}$ by a rectal probe connected to a temperature regulator controlling an overhead heating lamp. At the end of experiment, all animals were sacrificed and kidney and heart weights were then measured.

\section{Data analyses}

Single unit recording of renal sympathetic nerve activity was assessed by inserting a stainless steel electrode (12 $\mathrm{M} \Omega, 0.01$ Taper, A-M system, FL, USA) through surgically opened abdomen. The electrode holder was connected to a DAM-80 amplifier $(1,000 x, 500-1,000 \mathrm{~Hz}$ band pass filter; WPI, USA) and the BIOPAC MP 100 system (5,000 samples per second). Changes in renal nerve activity and heart rate were compared to mean arterial pressure following sodium nitroprusside or phenylephrine infusion to determine baroreceptor reflex sensitivity control of renal nerve activity (BSRA-S or BSRA-P) and heart rate (BSHR-S or BSHR-P). All offline data analyses were performed by the Acknowledge Software (BIOPAC).

All blood chemistry parameters were analyzed by the Khon Kaen University Hospital laboratory, with a blind control procedure. All data were expressed as mean \pm SEM. Statistical comparisons among groups $(\mathrm{p}<0.05)$ were performed by using one-way ANOVA and Duncan's Multi-Range tests (StatMost ver. 3.6 software, DataMost, USA).

\section{Results}

At 7-8 weeks of age, body, kidney, heart, kidney to body, and heart to body weights were not significantly different among the experimental groups (Table 1). Plasma sodium, plasma potassium, plasma creatinine, blood urea nitrogen, fasting blood sugar, and hematocrit were not significantly different among the groups (Table 2). Compared to CW, plasma insulin levels in CG groups were significantly higher, and captopril treatment eliminated this difference. Plasma insulin levels were not significantly different between TDW and TDG, but they were significantly higher than those in CW (about 2 time) and CG groups. In contrast to CG, captopril treatment markedly increased plasma insulin in TDW and TDG and moderately increased it in CW.

Mean arterial pressures were not significantly different among the groups, and they were significantly decreased by captopril treatment in all groups except TDG (Fig. 1). Heart rates were not significantly different among groups (Fig. 2). PE-induced baroreflex control of heart rate was not significantly different among control groups. In the TD groups, heart rate baroreflex was significantly impaired by glucose treatment, but this was returned to control levels by captopril treatment (Fig. 3). Captopril treatment did not alter these responses in the 
Table 1 Body (BW), kidney (KW), and heart (HW) weights in experimental groups

\begin{tabular}{cccccc}
\hline Treatment & BW (g) & HW (g) & KW (g) & HW/BW (\%) & KW/BW (\%) \\
\hline CW $(\mathbf{n}=7)$ & $183 \pm 2$ & $0.66 \pm 0.01$ & $0.69 \pm 0.01$ & $0.36 \pm 0.01$ & $0.38 \pm 0.01$ \\
CW+C (n=7) & $183 \pm 3$ & $0.70 \pm 0.02$ & $0.78 \pm 0.02$ & $0.38 \pm 0.01$ & $0.43 \pm 0.01$ \\
CG $(\mathbf{n}=7)$ & $182 \pm 3$ & $0.73 \pm 0.03$ & $0.78 \pm 0.04$ & $0.40 \pm 0.02$ & $0.43 \pm 0.02$ \\
CG+C (n=7) & $188 \pm 2$ & $0.84 \pm 0.02$ & $0.93 \pm 0.01$ & $0.45 \pm 0.01$ & $0.50 \pm 0.01$ \\
TDW $(\mathbf{n}=7)$ & $185 \pm 3$ & $0.72 \pm 0.02$ & $0.75 \pm 0.02$ & $0.39 \pm 0.01$ & $0.41 \pm 0.01$ \\
TDW+C (n=7) & $181 \pm 4$ & $0.71 \pm 0.03$ & $0.76 \pm 0.02$ & $0.39 \pm 0.01$ & $0.42 \pm 0.00$ \\
TDG (n=7) & $187 \pm 3$ & $0.81 \pm 0.03$ & $0.85 \pm 0.03$ & $0.43 \pm 0.01$ & $0.46 \pm 0.02$ \\
TDG+C $(\mathbf{n}=7)$ & $187 \pm 3$ & $0.79 \pm 0.04$ & $0.88 \pm 0.04$ & $0.42 \pm 0.02$ & $0.47 \pm 0.01$ \\
\hline
\end{tabular}

Data were mean \pm SEM. No significant difference among groups was noted (CW, control with water intake alone; CW+C, CW plus captopril treatment; CG, control with high sugar intake; CG+C, CG plus captopril treatment; TDW, perinatal taurine depletion with water intake alone ; TDW+C, TDW plus captopril treatment; TDG, perinatal taurine depletion with high sugar intake; TDG+C, TDG plus captopril treatment; TSW, perinatal taurine supplementation with water intake alone; TSW + C, TSW plus captopril treatment; TSG, perinatal taurine supplementation with high sugar intake; TSG+C, TSG plus captopril treatment).

other groups. A similar pattern was observed in responses to SNP (Fig. 4).

Baroreflex sensitivity control of renal sympathetic nerve activity following either PE (Fig. 5) or SNP (Fig. 6) decreased significantly only in TDG, and this was returned to control levels by captopril treatment. Interestingly, captopril treatment slightly decreased this measure in the other three groups (Fig. 6).

\section{Discussion}

Renin-angiotensin mechanisms underlie sugar-induced hypertension in many animal models [15]. High sugar intake (similar to that in the present study) induces renal dysregulation prior to its effects on hypertension and diabetes mellitus, and these changes are related to renin-angiotensin system overactivity [16]. The present data indicate that the renin-angiotensin system contributes to the blunted baroreflex control of arterial pressure in perinatal taurine-depleted adult female rats on a high sugar diet. In perinatal taurine-depleted adult male rats [10], high sugar intake increases sympathetic nerve activity and blunts baroreflex sensitivity, but the interplay of the renin-angiotensin system in this male model has not been tested.

The sympathetic nerves stimulate renin release from juxtraglomerular cells of the kidneys. Further, angiotensin II can activate the sympathetic nerves and norepinephrine release in the periphery and act in the anterior hypothalamus and rostral ventrolateral medulla $[17,18]$. Thus, the linkage between sympathetic nervous system, norepinephrine release, and the renin-angiotensin system can contribute to the genesis of many disorders via complex interactions. In spontaneously hypertensive rats, lifetime treatment with captopril prevents hypertension in adult life [7], but this treatment does not block dietary salt-sensitive hypertension in these animals. The salt-sensitive hypertension is related to sympathetic nervous system overactivity [6]. In sugarinduced hypertension, both the sympathetic nervous system and the renin-angiotensin system appear to play a major role. While the renin-angiotensin system may be the primary contributor to renal salt and water retention during the early phase of hypertension, both the sympathetic nervous and renin-angiotensin systems contribute importantly to sustained hypertension $[15,19,20]$.

Sugar-induced hypertension involves not only the sympathetic nervous system and the renin-angiotensin overactivity, but also hyperinsulinemia and insulin resistance [21]. The present findings confirm our previous reports that high sugar intake does not induce hyperglycemia and glucose intolerance in either control or perinatal taurine depleted female or male rats [9], but the

Table 2 General blood chemistry in experimental groups

\begin{tabular}{|c|c|c|c|c|c|c|c|}
\hline Treatment & $\mathrm{Na}(\mathrm{mEq} / \mathrm{l})$ & $\mathrm{K}(\mathrm{mEq} / \mathrm{l})$ & BUN (mg/dl) & $\mathrm{Cr}(\mathrm{mg} / \mathrm{dl})$ & FBS (mg/dl) & Insulin (pmol/l) & Hct (\%) \\
\hline CW $(n=7)$ & $142.9 \pm 2.9$ & $3.5 \pm 0.2$ & $20.6 \pm 1.3$ & $0.51 \pm 0.03$ & $102.4 \pm 3.1$ & $14.8 \pm 1.4$ & $48.1 \pm 1.2$ \\
\hline$C W+C(n=7)$ & $140.2 \pm 2.8$ & $3.9 \pm 0.2$ & $21.5 \pm 1.7$ & $0.55 \pm 0.02$ & $92 \pm 3.5$ & $26.0 \pm 3.3 *$ & $45.0 \pm 2.6$ \\
\hline CG $(n=7)$ & $141.5 \pm 1.8$ & $3.7 \pm 0.1$ & $22.8 \pm 1.8$ & $0.53 \pm 0.03$ & $103.2 \pm 3.8$ & $18.2 \pm 0.9 *$ & $44.8 \pm 2.1$ \\
\hline$C G+C(n=7)$ & $144.8 \pm 2.9$ & $3.7 \pm 0.2$ & $22.4 \pm 1.1$ & $0.54 \pm 0.03$ & $94.2 \pm 2.1$ & $15.7 \pm 1.5^{£}$ & $47.8 \pm 1.1$ \\
\hline TDW $(n=7)$ & $137.8 \pm 2.5$ & $3.6 \pm 0.2$ & $20.8 \pm 1.8$ & $0.53 \pm 0.03$ & $93.8 \pm 6.3$ & $26.8 \pm 1.5 *$ & $47.1 \pm 1.6$ \\
\hline $\mathrm{TDW}+\mathrm{C}(\mathrm{n}=7)$ & $144.1 \pm 2.7$ & $3.9 \pm 0.3$ & $22.0 \pm 1.6$ & $0.56 \pm 0.02$ & $94.1 \pm 2.9$ & $44.0 \pm 2.6^{*, \epsilon}$ & $48.8 \pm 1.7$ \\
\hline TDG $(n=7)$ & $136.3 \pm 2.9$ & $3.5 \pm 0.2$ & $21.9 \pm 1.2$ & $0.56 \pm 0.02$ & $92 \pm 4.7$ & $27.4 \pm 3.3 *$ & $44.1 \pm 2.3$ \\
\hline $\mathrm{TDG}+\mathrm{C}(\mathrm{n}=7)$ & $149.8 \pm 2.4$ & $3.6 \pm 0.1$ & $23.0 \pm 1.9$ & $0.55 \pm 0.03$ & $97.5 \pm 1.5$ & $73.8 \pm 7.9 *, \epsilon, \pm$ & $47 \pm 1.9$ \\
\hline
\end{tabular}

Data were mean \pm SEM ( ${ }^{*} \mathrm{P}<0.05$ compared to $\mathrm{CW},{ }^{\epsilon}$ to corresponding groups without captopril, ${ }^{£}$ to corresponding groups with captopril; Na, plasma sodium, $\mathrm{K}$, plasma potassium, BUN, blood urea nitrogen, $\mathrm{Cr}$, plasma creatinine, FBS, fasting blood sugar, Hct, hematocrit). For treatment abbreviations, see Table 1 


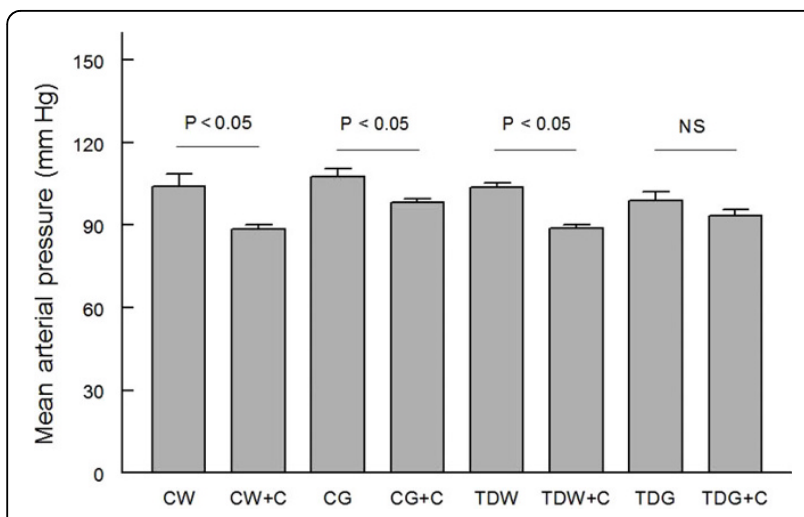

Figure 1 Comparison of resting mean arterial pressure among animal groups with different treatments. (CW, control with water intake alone; $\mathrm{CW}+\mathrm{C}$, CW plus captopril treatment; $\mathrm{CG}$, control with high sugar intake; CG+C, CG plus captopril treatment; TDW, perinatal taurine depletion with water intake alone; TDW+C, TDW plus captopril treatment; TDG, perinatal taurine depletion with high sugar intake; TDG+C, TDG plus captopril treatment).

present data indicate that although all animals displayed euglycemia, the insulin sensitivity of the groups were different (as estimated by the ratio of plasma glucose to insulin). During normal taurine exposure, high sugar intake (from weaning onward) slightly increases the plasma insulin concentration, but this is restored to control levels after captopril treatment. The finding that angiotensin II contributes to mild insulin resistance is supported by previous experiments [21]. Angiotensin II acts on insulin's target cells and alters post-receptor mechanisms to produce insulin resistance [22]. The insulin resistance may subsequently limit insulin clearance, resulting in increased plasma insulin. Renal metabolism and elimination of insulin is receptor-mediated $[23,24]$. Further, the renin-angiotensin system inhibits pancreatic, glucose-stimulated insulin secretion and biosynthesis and activates superoxide production that can induce pancreatic damage $[25,26]$. Thus, inhibition of

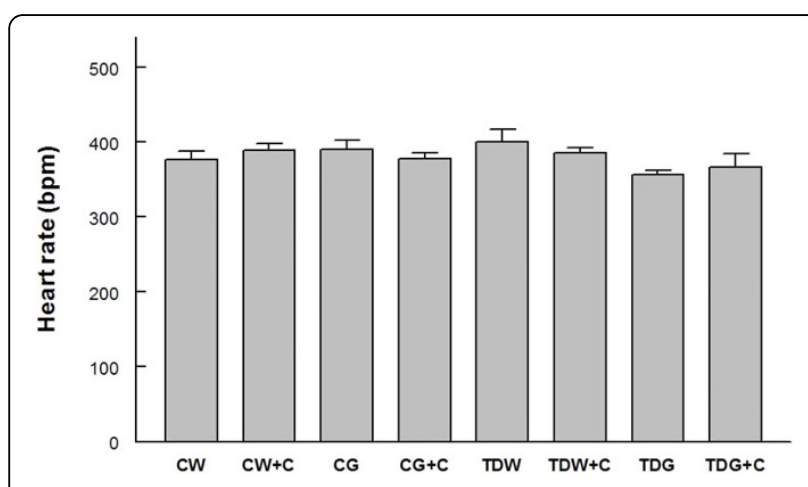

Figure 2 Comparison of resting heart rate among animal groups with different treatments. (for treatment abbreviations, see Figure 1).

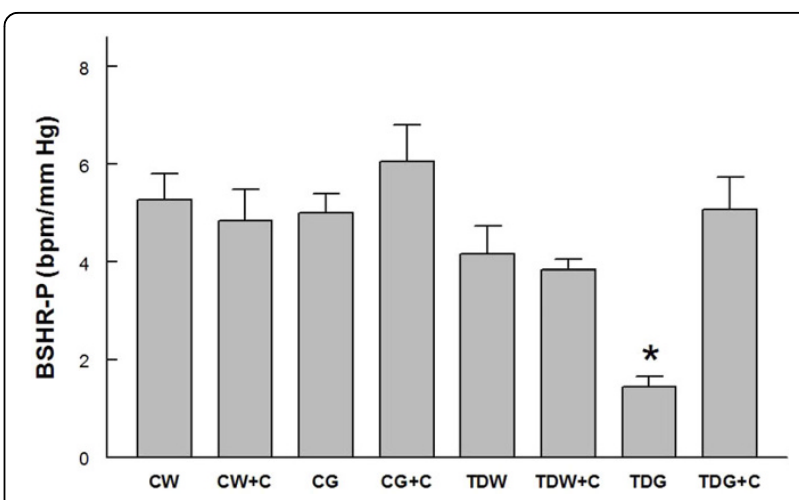

Figure 3 Baroreflex sensitivity control of heart rate tested by phenylephrine infusion (BSHR-P) in conscious rats. ${ }^{*} P<0.05$, compared to all other groups; for treatment abbreviations, see Figure 1).

the local renin-angiotensin system likely contributes to the insulin effects in the taurine depleted, sugar fed female rats in this study.

Perinatal taurine depletion increased plasma insulin concentration, but this was not further increased by high sugar intake. In contrast, captopril treatment greatly increased plasma insulin levels in taurinedepleted rats. The high sugar intake exacerbated this increase. In addition, fasting blood glucose was not significantly different among groups, suggesting that perinatal taurine depletion induces insulin resistance in female rats. It is well known that inhibition of the renin-angiotensin system increases insulin sensitivity and insulin secretion in humans and animals $[22,27]$. A marked rise in insulin resistance in $\mathrm{TDG}+\mathrm{C}$ more than $\mathrm{TDW}+\mathrm{C}$ (vs. normalization in $\mathrm{CG}+\mathrm{C}$ ) rats indicates an abnormal insulin/angiotensin II relationship in these animals that is exacerbated by the high sugar

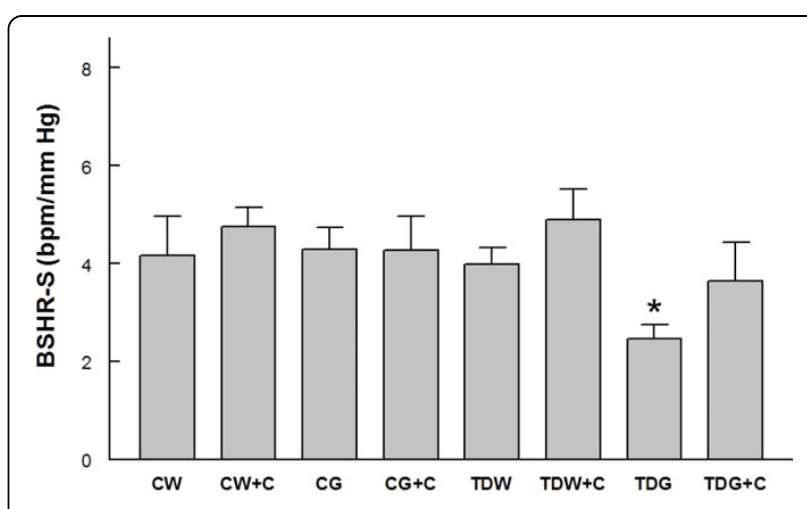

Figure 4 Baroreflex sensitivity control of heart rate tested by sodium nitroprusside infusion (BSHR-S) in conscious rats. ${ }^{*} P<$ 0.05, compared to all other groups; for treatment abbreviations, see Figure 1). 


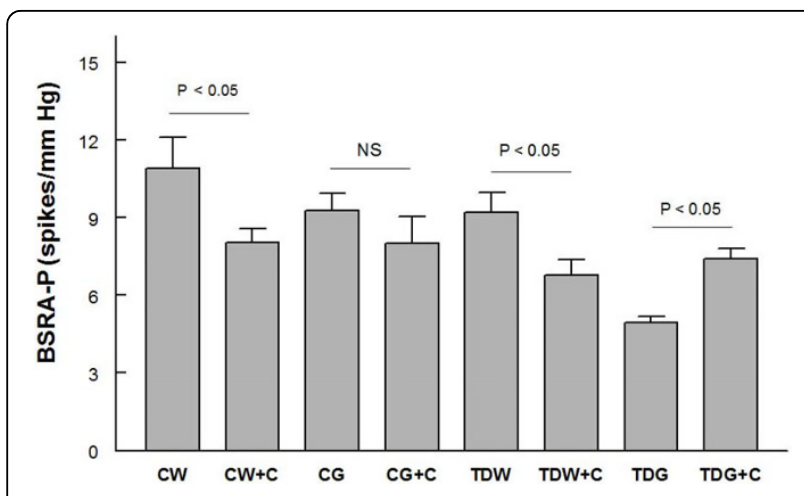

Figure 5 Baroreflex sensitivity control of renal sympathetic nerve activity tested by phenylephrine infusion (BSRA-P) in anesthetized rats. ${ }^{*} P<0.05$; for treatment abbreviations, see Figure 1).

intake. An abnormal insulin feedback inhibition may also contribute.

Inhibition of the renin-angiotensin system restored baroreflex sensitivity of the perinatal taurine-depleted rats to that of control groups, despite their hyperinsulinemia and insulin resistance. This suggests that reninangiotensin system overactivity primarily underlies this baroreflex depression. However, insulin enhances baroreflex sensitivity via the central nervous system $[22,28,29]$. TDG and TDW displayed similar decreased insulin sensitivity, but the baroreflex sensitivity was blunted only in TDG rats, suggesting that that blunted baroreflex is not due to insulin resistance alone. Whether a rise in plasma insulin level despite insulin resistance normalizes the baroreflex sensitivity in $\mathrm{TDG}+\mathrm{C}$ rats has to be further clarified.

In adult animals, taurine supplementation improves insulin resistance and hypertension in sugar-induced hypertension by inhibition of angiotensin II and its oxidative and other actions $[22,30,31]$. It also reduces the

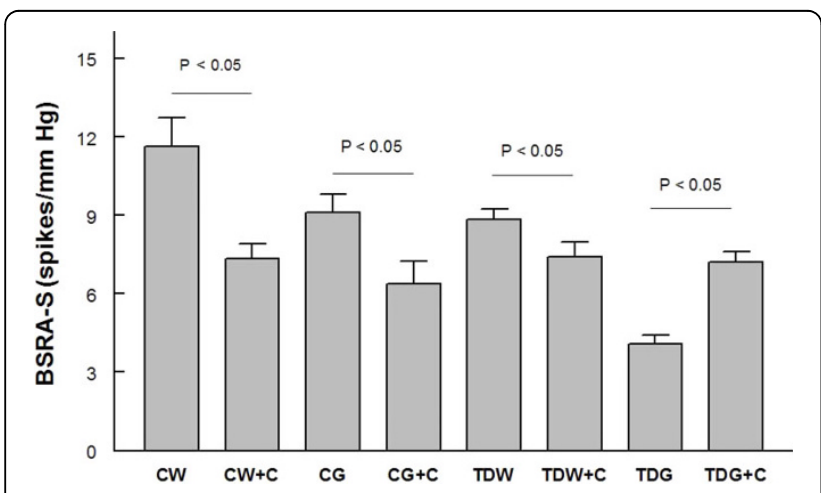

Figure 6 Baroreflex sensitivity control of renal sympathetic nerve activity tested by sodium nitroprusside infusion (BSRA-S) in anesthetized rats. ${ }^{*} P<0.05$; for treatment abbreviations, see Figure 1). cardiac hypertrophic effect of angiotensin II in animals $[32,33]$. In contrast, taurine deficiency in mature animals accelerates many adverse actions of angiotensin II on the heart, blood vessels, and kidney function [33,34]. In the kidney, taurine increases water and sodium excretion while angiotensin II acts in an opposite direction. In the medulla, angiotensin II injection increases taurine secretion [35]. Moreover, taurine stimulates, while angiotensin II inhibits, islet insulin secretion [36-38]. Together, these findings indicate that taurine and the renin-angiotensin system share many physiological roles in human bodies. The renin-angiotensin system is crucial for growth and development at perinatal life. Its deficiency produces organ damage and abnormalities in adults, especially related to the kidney $[4,5]$ and the nervous system [39]. Similarly, sufficient taurine exposure during a perinatal period leads to normal adult organ function [1]. The present data confirm these relationships. Perinatal taurine depletion may increase the renin-angiotensin system activity at early life and this change may lead to permanent effects that cannot be reversed by later taurine availability, i.e., the programming of renin-angiotensin system function in mature life is likely dictated by the perinatal exposure.

\section{Conclusion}

Perinatal taurine depletion does not alter baroreflex sensitivity but induces hyperinsulinemia and insulin resistance. High sugar intake blunts baroreflex function but does not modify the hyperinsulinemia or insulin resistance. When treated with captopril, the blunted baroreflex is completely normalized, but hyperinsulinemia and insulin resistance is exacerbated. Thus, the present data indicate that in perinatal taurine depleted adult, female rats, high sugar intake blunts baroreceptor reflexes, at least in part, via renin-angiotensin system overactivity, but insulin resistance does not appear to contribute to the blunted baroreflex.

\section{List of abbreviations used}

CW: control with water intake alone; $\mathrm{CW}+\mathrm{C}$ : CW plus captopril treatment; CG: control with high sugar intake; CG+C: CG plus captopril treatment; TDW: perinatal taurine depletion with water intake alone; TDW+C: TDW plus captopril treatment; TDG: perinatal taurine depletion with high sugar intake; TDG+C: TDG plus captopril treatment; BW: body weight; HW: heart weight; KW: kidney weight; SD: Sprague Dawley; i.p.: intraperitoneal; PE: phenylephrine; SNP: sodium nitroprusside; BSRA-S or BSRA-P: baroreceptor reflex sensitivity control of renal nerve activity tested by SNP or PE; BSHR-S or BSHR-P: baroreceptor reflex sensitivity control of heart rate tested by SNP or PE; SEM: standard error of means; BUN: blood urea nitrogen; FBS: fasting blood sugar; Hct: hematocrit; K: plasma potassium, Na, plasma sodium; Cr: plasma creatinine.

\section{Acknowledgements}

This article has been published as part of Journal of Biomedical Science Volume 17 Supplement 1, 2010: Proceedings of the 17th International Meeting of Taurine. The full contents of the supplement are available online at http://www.jbiomedsci.com/supplements/17/S1. 
This work is supported in part by grants from the Faculty of Medicine, Khon Kaen University, Khon Kaen 40002, Thailand and by the USA National Institutes of Health (NIH) grant AT 00477 (JMW).

\section{Author details}

${ }^{1}$ Department of Physiology Faculty of Medicine, Khon Kaen University, Khon Kaen 40002, Thailand. ${ }^{2}$ Department of Cell Biology, University of Alabama at Birmingham, AL 35294, USA.

\section{Authors' contributions}

1. Atcharaporn Thaeomor: research proposal preparation, data collection and analysis, article preparation

2. J. Michael Wyss: research consult, article preparation

3. Dusit Jirakulsomchok: research consult, article preparation

4. Sanya Roysommuti: research proposal design, data analysis, article preparation, correspondence

\section{Competing interests}

The authors declare that they have no competing interests.

Published: 24 August 2010

\section{References}

1. Sturman JA: Taurine in development. Physiol Rev 1993, 73:119-147.

2. Aerts $L$, Van Assche FA: Taurine and taurine-deficiency in the perinatal period. J Perinat Med 2002, 30:281-286.

3. Barker DJ, Eriksson JG, Forsen T, Osmond C: Fetal origins of adult disease: strength of effects and biological basis. Int J Epidemiol 2002, 31:1235-1239.

4. Guron G, Marcussen N, Nilsson A, Sundelin B, Friberg P: Postnatal time frame for renal vulnerability to enalapril in rats. J Am Soc Nephrol 1999, 10:1550-1560.

5. Guron G, Nilsson A, Nitescu N, Nielsen S, Sundelin B, Frokiaer J, et al: Mechanisms of impaired urinary concentrating ability in adult rats treated neonatally with enalapril. Acta Physiol Scand 1999, 165:103-112.

6. Wyss JM, Mozaffari MS, Roysommuti S: Contribution of the sympathetic nervous system to salt-sensitivity in lifetime captopril-treated spontaneously hypertensive rats. J Hypertens 1995, 13:1037-1042.

7. Wyss JM, Roysommuti S, King K, Kadisha I, Regan CP, Berecek KH: Saltinduced hypertension in normotensive spontaneously hypertensive rats. Hypertension 1994, 23:791-796.

8. Racasan S, Braam B, van der Giezen DM, Goldschmeding R, Boer P, Koomans HA, et al: Perinatal L-arginine and antioxidant supplements reduce adult blood pressure in spontaneously hypertensive rats. Hypertension 2004, 44:83-88.

9. Roysommuti S, Suwanich A, Lerdweeraphon W, Thaeomor A, Jirakulsomchok D, Wyss JM: Sex dependent effects of perinatal taurine exposure on the arterial pressure control in adult offspring. Adv Exp Med Biol 2009, 643:135-144

10. Roysommuti $S$, Suwanich A, Jirakulsomchok D, Wyss JM: Perinatal taurine depletion increases susceptibility to adult sugar-induced hypertension in rats. Adv Exp Med Biol 2009, 643:123-133.

11. Roysommuti $S$, Malila P, Jikulsomchok $S$, Jirakulsomchok D, Wyss JM: Perinatal taurine status influences renal hemodynamics in adult conscious rats. FASEBJ 2004, 18:A292-A293.

12. Han X, Budreau AM, Chesney RW: The taurine transporter gene and its role in renal development. Amino Acids 2000, 19:499-507.

13. Huang DY, Boini KM, Lang PA, Grahammer F, Duszenko M, Heller-Stilb B, et al: Impaired ability to increase water excretion in mice lacking the taurine transporter gene TAUT. Pflugers Arch 2006, 451:668-677.

14. Roysommuti S, Lerdweeraphon W, Malila P, Jirakulsomchok D, Wyss JM: Perinatal taurine alters arterial pressure control and renal function in adult offspring. Adv Exp Med Biol 2009, 643:145-156.

15. Farah V, Elased KM, Morris M: Genetic and dietary interactions: role of angiotensin AT1a receptors in response to a high-fructose diet. Am J Physiol Heart Circ Physiol 2007, 293:H1083-H1089.

16. Roysommuti $S$, Khongnakha T, Jirakulsomchok D, Wyss JM: Excess dietary glucose alters renal function before increasing arterial pressure and inducing insulin resistance. Am J Hypertens 2002, 15:773-779.
17. Allen AM, O'Callaghan EL, Chen D, Bassi JK: Central neural regulation of cardiovascular function by Angiotensin: a focus on the rostral ventrolateral medulla. Neuroendocrinology 2009, 89:361-369.

18. DiBona GF: Peripheral and central interactions between the reninangiotensin system and the renal sympathetic nerves in control of renal function. Ann N Y Acad Sci 2001, 940:395-406.

19. Preuss $\mathrm{HG}$, Memon S, Dadgar A, Gongwei J: Effects of high sugar diets on renal fluid, electrolyte and mineral handling in rats: relationship to blood pressure. J Am Coll Nutr 1994, 13:73-82.

20. Mayer MA, Hocht C, Gironacci M, Opezzo JA, Taira CA, Fernandez BE, et al: Hypothalamic angiotensinergic-noradrenergic systems interaction in fructose induced hypertension. Regul Pept 2008, 146:38-45.

21. Tran $L T$, Yuen VG, McNeill JH: The fructose-fed rat: a review on the mechanisms of fructose-induced insulin resistance and hypertension. Mol Cell Biochem 2009, 332:145-159.

22. Olivares-Reyes JA, Arellano-Plancarte A, Castillo-Hernandez JR: Angiotensin II and the development of insulin resistance: implications for diabetes. Mol Cell Endocrinol 2009, 302:128-139.

23. Meshkani R, Adeli K: Hepatic insulin resistance, metabolic syndrome and cardiovascular disease. Clin Biochem 2009, 42:1331-1346.

24. Rabkin R, Ryan MP, Duckworth WC: The renal metabolism of insulin. Diabetologia 1984, 27:351-357.

25. Chu KY, Lau T, Carlsson PO, Leung PS: Angiotensin II type 1 receptor blockade improves beta-cell function and glucose tolerance in a mouse model of type 2 diabetes. Diabetes 2006, 55:367-374.

26. Lau T, Carlsson PO, Leung PS: Evidence for a local angiotensin-generating system and dose-dependent inhibition of glucose-stimulated insulin release by angiotensin II in isolated pancreatic islets. Diabetologia 2004, 47:240-248.

27. Lastra-Lastra G, Sowers JR, Restrepo-Erazo K, Manrique-Acevedo C, LastraGonzalez G: Role of aldosterone and angiotensin II in insulin resistance: an update. Clin Endocrinol (Oxf) 2009, 71:1-6.

28. Okada M, Bunag RD: Insulin acts centrally to enhance reflex tachycardia in conscious rats. Am J Physiol 1994, 266:R481-R486.

29. Pricher MP, Freeman KL, Brooks VL: Insulin in the brain increases gain of baroreflex control of heart rate and lumbar sympathetic nerve activity. Hypertension 2008, 51:514-520.

30. Harada H, Tsujino T, Watari $Y$, Nonaka H, Emoto N, Yokoyama M: Oral taurine supplementation prevents fructose-induced hypertension in rats. Heart Vessels 2004, 19:132-136.

31. Nandhini AT, Thirunavukkarasu V, Anuradha CV: Potential role of kinins in the effects of taurine in high-fructose-fed rats. Can J Physiol Pharmacol 2004, 82:1-8.

32. Azuma M, Takahashi K, Fukuda T, Ohyabu Y, Yamamoto I, Kim S, et al: Taurine attenuates hypertrophy induced by angiotensin II in cultured neonatal rat cardiac myocytes. Eur J Pharmacol 2000, 403:181-188.

33. Schaffer SW, Lombardini JB, Azuma J: Interaction between the actions of taurine and angiotensin II. Amino Acids 2000, 18:305-318.

34. Cruz Cl, Ruiz-Torres P, del Moral RG, Rodriguez-Puyol M, Rodriguez-Puyol D: Age-related progressive renal fibrosis in rats and its prevention with ACE inhibitors and taurine. Am J Physiol Renal Physiol 2000, 278:F122-F129.

35. Zhu DN, Moriguchi A, Mikami H, Higaki J, Ogihara T: Central amino acids mediate cardiovascular response to angiotensin II in the rat. Brain Res Bull 1998, 45:89-197.

36. El Idrissi A, Boukarrou L, L'Amoreaux W: Taurine supplementation and pancreatic remodeling. Adv Exp Med Biol 2009, 643:353-358.

37. Ribeiro RA, Bonfleur ML, Amaral AG, Vanzela EC, Rocco SA, Boschero AC, et al: Taurine supplementation enhances nutrient-induced insulin secretion in pancreatic mice islets. Diabetes Metab Res Rev 2009, 25:370-379.

38. Leung PS: The physiology of a local renin-angiotensin system in the pancreas. J Physiol 2007, 580:31-37.

39. Sanchez SI, Arce ME, Fuentes LB, Ciuffo GM: Prenatal blockade of Ang II receptors affects neonatal rat hindbrain structure and receptor localization. Exp Neurol 2009, 220:246-254.

doi:10.1186/1423-0127-17-S1-S30

Cite this article as: Thaeomor et al:: High sugar intake via the reninangiotensin system blunts the baroreceptor reflex in adult rats that were perinatally depleted of taurine. Journal of Biomedical Science 2010 17(Suppl 1):S30. 